\title{
David Page
}

The 2011 March of Dimes Prize in

Developmental Biology has been jointly awarded to Patricia Jacobs, of Southampton University Medical School and the Wessex Regional Genetics Laboratory, and to David Page, of the Whitehead Institute, Massachusetts Institute of Technology and Howard Hughes Medical Institute, for their pioneering research on the $\mathrm{X}$ and $\mathrm{Y}$ chromosomes. The prize recognizes researchers whose work has contributed to our understanding of the science that underlies birth defects. We talked to the winners about their achievements, the impact these have had on human health and where their current research is taking them. This month's interview is with David Page, who spoke to Louisa Flintoft. The interview with Patricia Jacobs appeared in our June issue.

\section{How did you become interested in mammalian sex chromosomes?} I started out in the summer of 1979 working with David Botstein and Ray White on the idea of making a genetic linkage map of the human genome. I think I was the first student to work on what would become the Human Genome Project. My task was to generate restriction fragment length polymorphisms (RFLPs) from throughout the human genome and bring them together to make a map. I was picking DNA fragments at random from a human genome library to turn them into hybridization probes. One of the first probes that I picked came from what would prove to be a site of homology between the X and Y chromosomes, and that essentially determined the course of my career. I then started to wonder what you could do with probes from the $\mathrm{X}$ and Y chromosomes. At the first scientific meeting I ever attended, Albert de la Chappelle - who had described the first case of human XX males - walked by my poster. Soon we were collaborating and I started thinking about sex chromosome disorders and how you could study them using DNA probes.

\section{What insights did these studies bring?} By the mid 1980s, it was becoming clear that whether an embryo was anatomically masculinized or feminized depends on a single part of the Y chromosome something that Patricia Jacobs had pointed to earlier but that had been difficult to establish using purely cytogenetic methods. It became a tractable problem only with the introduction of DNA probe-based deletion mapping of the Y chromosome. Ironically, I was working on the only human chromosome

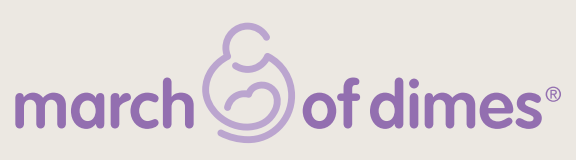

clone- and PCR-marker-based maps of the Y chromosome, which was a big advance. The 1990s were a time of becoming convinced that there were many more genes on the $\mathrm{Y}$ chromosome than anyone had supposed, that most of those genes were involved in sperm production and that $\mathrm{Y}$ deletions were a frequent cause of spermatogenic failure. But we still didn't have a good map of the most critical regions, which turned about to be structural nightmares.

In the late 1990s, we partnered with the genome-sequencing centre at Washington University (St. Louis, Missouri, USA) to sequence the human Y chromosome. That opened up a new phase that revealed completely unexpected aspects, including the near-perfect massive palindromes that dominate the landscape of the long arm of the Y chromosome. What was exciting about this realization was that it also gave an explanation for the Y chromosome's propensity for deletion - homologous recombination was the answer. It was emerging as a phenomenon across the genome, but it looked as if the Y chromosome was perhaps more dominated by this feature than any other chromosome.

\section{What has your work meant for patients?}

As we entered the field in early 1990s, we began to realize how little solid knowledge there was about the causes of spermatogenic failure. One of the significant upshots of our work with respect to $\mathrm{Y}$ chromosome deletions and spermatogenic failure was to bring genetic thinking to a prominent place in the consideration of male infertility. Many labs around the world joined in with this task.

More specifically, the $\mathrm{Y}$ deletions that we, and others, discovered account for perhaps $10-15 \%$ of the more severe cases of spermatogenic failure. So, for many couples this work brought diagnoses where they didn't exist before, which have proved important in making clinical decisions. For men who carry the most common Y deletion, even if they produce very few sperm and even if there are none in the ejaculate, sperm can often be retrieved from a testis biopsy. But other deletions on the $\mathrm{Y}$ chromosome appear to be completely incompatible with spermatogenesis, so couples must then consider other options. 